UWThPh-2000-14

ESI-864-2000

math-ph/0004006

April 2, 2000

\title{
Thermal Correlators of Anyons in Two Dimensions
}

\author{
N. Ilieva*, H. Narnhofer and W. Thirring \\ Institut für Theoretische Physik \\ Universität Wien \\ and \\ Erwin Schrödinger International Institute \\ for Mathematical Physics
}

\begin{abstract}
The anyon fields have trivial $\alpha$-commutator for $\alpha$ not integer. For integer $\alpha$ the commutators become temperature-dependent operator valued distributions. The $n$-point functions do not factorize as for quasifree states.
\end{abstract}

PACS Numbers: 03.70.+k, 11.10.Kk, 11.10.Wx, 71.10.Pm

Keywords: two-dimensional models, fractional statistics, KMS states, $n$-point functions

* On leave from Institute for Nuclear Research and Nuclear Energy, Bulgarian Academy of Sciences, Boul.Tzarigradsko Chaussee 72, 1784 Sofia, Bulgaria 


\section{Introduction}

The problem of the Bose-Fermi duality in two-dimensional space-time has a long history. Since the pioneering works in the thirties [1, 2] and till now, various of its aspects, conceptual, as well as technical, remain attractive due to the ambitious idea to extend this remarkable feature also beyond one space dimension. In recent papers [3, 田, starting with bare fermions which form the $C^{*}$-algebra $\mathcal{A}=\operatorname{CAR}(\mathbf{R})$, we have constructed a chain of algebraic inclusions to substanciate our understanding of this phenomenon:

$$
\mathrm{CAR}(\text { bare }) \subset \pi_{\beta}(\mathcal{A})^{\prime \prime} \supset \mathcal{A}_{c} \subset \overline{\mathcal{A}}_{c} \subset \bar{\pi}_{\beta}\left(\overline{\mathcal{A}}_{c}\right)^{\prime \prime} \supset \operatorname{CAR}(\text { dressed })
$$

To get the first extension, we note that the shift $\tau_{t}$ is an automorphism of $\mathcal{A}$ which has KMS-states $\omega_{\beta}$ and associated representations $\pi_{\beta}$. One then identifies in $\pi_{\beta}(\mathcal{A})^{\prime \prime}$ bosonic modes - the currents, which form the current algebra $\mathcal{A}_{c}$ with a $\beta$-independent structure for $0<\beta<\infty$. The crucial ingredient needed at this step is the appropriately chosen state. We choose the KMS-state which is unique for the shift over the CAR algebra. Another possibility would be to chose the Dirac vacuum. This is what has originally been done in [1], 2], in order to achieve stability for a fermionic system, and recovered later by Mattis and Lieb [5] in the context of the Luttinger model.

The essential result is the appearance of an anomalous (Schwinger) term in the quantum current commutator

$$
\left[j(x), j\left(x^{\prime}\right)\right]=-\frac{i}{2 \pi} \delta^{\prime}\left(x-x^{\prime}\right)
$$

based on which Mandelstam proposed $e^{i 2 \pi \int_{-\infty}^{x} j(y) d y}$ as a fermion field [6]. In this note we construct the anyonic fields

$$
\Psi_{\alpha}(x) \simeq e^{i 2 \pi \sqrt{\alpha} \int_{-\infty}^{x} j(y) d y}
$$

through their action on the bosonic current and study them as operators in a Hilbert space by exhibiting their n-point function in a $\tau$-KMS state $\omega, \tau$ being the shift automorphism. This corresponds to taking the crossed product [7, 8] of $\mathcal{A}_{c}$ with an outer automorphism [9] or, equivalently, augmenting $\mathcal{A}_{c}$ by an unitary operator $U_{\alpha}=e^{i 2 \pi \sqrt{\alpha} j_{\varphi_{\eta}}}\left(\alpha \in \mathbf{R}^{+}\right.$, and $j_{\varphi_{\eta}}$ being the smeared current with an appropriately chosen test function $\varphi_{\eta}$, which converges to a constant for $\eta \rightarrow 0, x \rightarrow \infty)$ to $\overline{\mathcal{A}}_{c}$. Then one discovers in $\bar{\pi}_{\beta}\left(\overline{\mathcal{A}}_{c}\right)^{\prime \prime}$ anyonic modes which satisfy Heisenberg's Urgleichung [10] in a distributional sense

$$
\frac{1}{i} \frac{\partial}{\partial x} \Psi_{\alpha}(x)=\pi \sqrt{\alpha}\left[j(x), \Psi_{\alpha}(x)\right]_{\alpha} .
$$

Thus we do not introduce the anyons as fields with deformed commutation relations as e.g. in [11] but we obtain these relations.

The important point here is that the Hilbert space $\overline{\mathcal{H}}_{\beta}$ assumes a sectorial structure, being for fixed $\alpha$ a countable orthogonal sum of sectors with $n$ particles created by $U_{\alpha}$

$$
\overline{\mathcal{H}}_{\beta}=\oplus \overline{\mathcal{H}}_{\beta}^{n}, \quad \overline{\mathcal{H}}_{\beta}^{n}=\mathcal{A}_{c} \prod_{i=1}^{n} \Psi_{\eta}\left(x_{i}\right)|\Omega\rangle
$$

Here the following is to be observed: 
1. $\Psi_{\alpha}$ as in (1.3) has an infrared and an ultraviolet problem. The infrared divergence actually shows that admitting the (smeared) step function as a test function, one creates new elements in the field algebra which lead to orthogonal sectors in a larger Hilbert space, Eq.(1.4). The ultraviolet divergence does not lead out of $\bar{\pi}_{\beta}$ if we smear $j(y)$ over a region of size $\eta$ to get $\Psi_{\alpha, \eta}$ and consider the renormalized field

$$
\lim _{\eta \rightarrow 0^{+}} c_{\alpha}(\eta) \int d x f(x) \Psi_{\alpha, \eta}(x)=\Psi_{\alpha}(f)
$$

with a suitable $c_{\alpha}(\eta)$. This limit exists in a strong sense and $\Psi(f)$ has finite n-point functions.

2. When the statistical parameter $\alpha$ is an integer, two special families of such renormalized operators are distinguished: for odd $\alpha$ 's we get fermions and for even $\alpha$ 's bosons. However, only the field $\Psi_{1}$ turns out to be a canonical Fermi field,

$$
\left[\Psi_{1}^{*}(x), \Psi_{1}\left(x^{\prime}\right)\right]_{+}=\delta\left(x-x^{\prime}\right)
$$

with an n-point function of the familiar determinant form. $\Psi_{2}$ is a non-canonical Bose field, whose commutator is not a $c$-number

$$
\left[\Psi_{2}^{*}(x), \Psi_{2}\left(x^{\prime}\right)\right]=\delta^{\prime}\left(x-x^{\prime}\right)+i j(x) \delta\left(x-x^{\prime}\right) .
$$

Similarly, the operator $\Psi_{3}$ describes a non-canonical (unbounded) Fermi field. For $\alpha \notin \mathbf{Z}$ the anyonic commutator vanishes.

The algebraic chain (1.1) means that the dressed fermions obtained for special values of $\alpha$ can be constructed either from bare fermions or directly from the current algebra, so in this case it cannot be decided whether fermions or bosons are more fundamental. Moreover, we shall argue that the fermions at both "ends" are actually equivalent, so the corresponding algebras do coincide. To make this statement precise, the correlation functions arrising in both cases have to be compared and this will be done subsequently.

\section{The bosonic algebra and its states}

Consider the smeared currents $j(f)=\int j(x) f(x) d x$ with real-valued functions $f(x) \in \mathcal{C}_{0}^{\infty}$, so that neither infrared nor ultraviolet problems occur. $f$ 's form a real pre-Hilbert space to be defined subsequently. For the bosonic algebra built by the Weyl operators $e^{i j(f)}$, (1.2) is replaced by the multiplication law for the unitaries

$$
e^{i j(f)} e^{i j(g)}=e^{\frac{i}{2} \sigma(g, f)} e^{i j(f+g)},
$$

with a symplectic form

$$
\sigma(f, g)=\frac{1}{4 \pi} \int_{-\infty}^{\infty} d x\left(f^{\prime}(x) g(x)-f(x) g^{\prime}(x)\right) .
$$


As an integral kernel the symplectic form reads $\sigma(x-y)=\delta^{\prime}(x-y) / 2 \pi$. Therefore the bosonic algebra satisfies the requirement of a local field theory.

We shall be interested in states that are invariant under a time evolution $\tau_{t}$, in particular — KMS states. An equilibrium state of a quantum system at a finite temperature $T=\beta^{-1}$ is characterized by the KMS-condition

$$
\omega_{\beta}\left(\tau_{t}(A) B\right)=\omega_{\beta}\left(B \tau_{t+i \beta} A\right)
$$

with the time evolution $\tau_{t}$ as an automorphism of the algebra of observables analytically continued for imaginary times. In the bosonic (current) algebra $\mathcal{A}_{c}$ this role is played by the shift

$$
\tau_{t} j(f(x))=j(f(x-t)) .
$$

The time-invariant states are in general defined by the two-point function

$$
\omega(j(f) j(g))=\int d x d y w(x-y) f(x) g(y),
$$

so for the $\tau$-KMS state at a temperature $\beta=\pi$ the kernel $w(x-y)$ reads

$$
w(x-y)=-\lim _{\varepsilon \rightarrow 0^{+}} \frac{1}{(2 \pi)^{2} \operatorname{sh}^{2}(x-y-i \varepsilon)} .
$$

If we want to avoid distributions being involved into the calculations, we can consider the smeared functions

$$
f_{\eta}(x)=\int f(y) h_{\eta}(x-y) d y, \quad j\left(f_{\eta}\right)=j_{\eta}(f),
$$

with some $h_{\eta} \in \mathcal{C}_{0}^{\infty}$. This leads to a new symplectic form

$$
\begin{gathered}
\sigma\left(f_{\eta}, g_{\eta}\right) \equiv \sigma_{\eta}(f, g), \\
\sigma_{\eta}(x-y)=\frac{1}{2 \pi} \int \delta^{\prime}\left(z-z^{\prime}\right) h_{\eta}(z-x) h_{\eta}\left(z^{\prime}-y\right) d z d z^{\prime} \\
=\frac{1}{2 \pi} \int h_{\eta}(z-x) h_{\eta}^{\prime}(z-y) d z .
\end{gathered}
$$

In this case $\varepsilon$ of (2.3) need not be finite but just indicates which distribution is understood. We shall henceforth omit keep writing $\lim _{\varepsilon \rightarrow 0}$.

$\mathcal{C}_{0}^{\infty}$ functions and functions with steps are mapped by the smearing into $\mathcal{C}_{0}^{\infty}$ functions. Therefore the bosonic algebra remains unchanged. The essential point is that the fields $j_{\eta}(x)$ are not local any more in the sense that they do not commute if they are spatially separated. On the other hand, we gain that they become (unbounded) operators and not only operator-valued distributions. Their time evolution is given by

$$
\tau_{t} j_{\eta}(x)=j_{\eta}(x+t) .
$$

Again we can express the state by

$$
\omega\left(j\left(f_{\eta}\right) j\left(g_{\eta}\right)\right)=\omega\left(j_{\eta}(f) j_{\eta}(g)\right):=\langle f \mid g\rangle_{\eta}
$$


where the corresponding integral kernel

$$
w_{\eta}(x-y)=\int w\left(z-z^{\prime}\right) h_{\eta}(x-z) h_{\eta}\left(y-z^{\prime}\right) d z d z^{\prime}
$$

satisfies

$$
w_{\eta}(x-y)-w_{\eta}(y-x)=\sigma_{\eta}(x-y) .
$$

The expectation of the Weyl operators is given by

$$
\omega\left(e^{i j_{\eta}(f)}\right)=e^{-\frac{1}{2}\langle f \mid f\rangle_{\eta}} .
$$

Eqs.(2.1),(2.6) imply

$$
\omega\left(\prod_{k} e^{i j_{\eta}\left(f_{k}\right)}\right)=\exp \left\{-\frac{1}{2}\left[\sum_{k}\left\langle f_{k} \mid f_{k}\right\rangle_{\eta}+2 \sum_{k<m}\left\langle f_{k} \mid f_{m}\right\rangle_{\eta}\right]\right\} .
$$

If $\lim _{\eta \rightarrow 0} h_{\eta}(x)=\delta(x)$, then $\lim _{\eta \rightarrow 0}\langle f \mid g\rangle_{\eta}=\langle f \mid g\rangle$ and the operator $j_{\eta}(x)$ approaches the distribution $j(x)$.

With the scalar product $\langle f \mid g\rangle$ the one-particle Hilbert space $h$ is defined as the closure of the pre-Hilbert space $\mathcal{C}_{0}^{\infty}$, that determines the bosonic von Neumann algebra $\bar{\pi}_{\beta}(\mathcal{A})^{\prime \prime}=: \mathcal{A}_{B}$. Of course, $\langle f \mid g\rangle_{\eta}$ defines the same Hilbert space, but infrared and ultraviolet divergencies are kept apart.

\section{The extension to anyons}

The field operators we wish to construct in the spirit of Mandelstam are of the form $\Psi_{\alpha}(x) \simeq e^{i 2 \pi \sqrt{\alpha} \int_{-\infty}^{x} j(y) d y}$, so they are defined by the function $F_{x}(y)=2 \pi \Theta(x-y)$, whereas the functions from $h$ have to vanish for $x \rightarrow \pm \infty$. The symplectic form (2.2) is defined for functions that tend to a constant, however they cannot be reached as limits of functions from $h$. For instance,

$$
F_{x, \delta}(y)=\Theta(x-y)-\Theta(x-y-\delta), \quad F_{x, \delta, \eta} \in \mathcal{C}_{0}^{\infty} .
$$

does not work, since $\sigma\left(F_{x, \delta}, F_{x^{\prime}, \delta^{\prime}}\right)$ depends on the order in which the limits $\delta, \delta^{\prime} \rightarrow \infty$ are taken and only for $\delta=\delta^{\prime} \rightarrow \infty$ we get the desired result $i \operatorname{sgn}\left(x-x^{\prime}\right)$. Since this appears in the $c$-number part, in no representation can $j\left(F_{x, \delta}\right)$ converge strongly. Nevertheless, for functions with the same (nontrivial) asymptotics at, say, $x \rightarrow \infty$ and whose difference $\in h$ one can succeed in getting the expectation values as limits.

The desired extension of the algebra $\mathcal{A}_{B}$ can be achieved in two equivalent ways. The one-particle Hilbert space can be enlarged [12, [3] allowing also for (appropriately smeared) step functions, e.g. $F_{x}^{\alpha}=\sqrt{\alpha} F_{x}$, such that only $F^{\prime} \in \mathcal{C}_{0}^{\infty}$. For the enlarged algebra the symplectic form (2.2) is kept. If the localization of the current is given by support $F^{\prime}$, then fields that are localized in different regions satisfy the following exchange relation ( $\alpha$-commutator)

$$
e^{i j\left(F^{\alpha}\right)} e^{-i j\left(G^{\alpha}\right)}=e^{i \pi \alpha} e^{-i j\left(G^{\alpha}\right)} e^{i j\left(F^{\alpha}\right)}, \quad \operatorname{supp} F^{\prime}<\operatorname{supp} G^{\prime}
$$




$$
\begin{gathered}
\lim _{x \rightarrow-\infty} F(x)=\lim _{x \rightarrow-\infty} G(x)=2 \pi, \\
\lim _{x \rightarrow \infty} F(x)=\lim _{x \rightarrow \infty} G(x)=0 .
\end{gathered}
$$

The same algebra can be obtained by the automorphism $\gamma_{F^{\prime}}$ of the initial bosonic algebra $\mathcal{A}_{c}$

$$
\gamma_{F^{\prime}} e^{i j(f)}=e^{i \int F^{\prime}(x) f(x) d x} e^{i j(f)} .
$$

This automorphism is not inner but allows the construction of a crossed product in which $\gamma_{F^{\prime}}$ is implemented by $e^{i j(F)}$ (compare [9]). A given quasifree automorphism $\rho$ on the initial algebra can be uniquely extended to an automorphism on the enlarged algebra provided $\gamma_{F^{\prime}} \rho \gamma_{F^{\prime}}^{-1} \rho^{-1}$ is an inner automorphism of $\mathcal{A}_{c}$. For instance, $\tau_{t}$ is extendable and acts again as a shift on $j(F)$. Also a state on the bosonic algebra can be extended according to

$$
\omega\left(e^{i j(F)}\right)=0 \quad \text { if } \lim _{x \rightarrow-\infty} F(x) \neq 0 .
$$

For higher products we combine

$$
\omega\left(e^{i j(f)} e^{i j(F)} e^{-i j(G)} e^{-i j(g)}\right)=\omega\left(e^{i j(f)} e^{i j(F-G)} e^{-i j(g)}\right) e^{i \sigma(F, G) / 2} .
$$

The operator $e^{i j(F-G)} \in \mathcal{A}_{B}$ and the expectation value is well defined.

As already mentioned, whereas $e^{i j(F)}$ itself cannot be obtained as a limit of operators in $\mathcal{A}_{B}$, the situation with the expectation value is different. We concentrate on the currents $j_{\eta}\left(F_{x}\right)$, where $\eta$ indicates that we have smeared as in $(2.4),(2.5)$ and choose the test function (3.1). With this, we get for $\delta>|x-\bar{x}|+\left|\operatorname{supp} h_{\eta}\right|$

$$
\begin{aligned}
& e^{i j_{\eta}\left(F_{x, \delta}^{\alpha}\right)} e^{-i j_{\eta}\left(F_{\bar{x}, \delta}^{\alpha}\right)}=e^{i j_{\eta}\left(F_{x, \delta}^{\alpha}-F_{\bar{x}, \delta}^{\alpha}\right)+i \alpha \sigma_{\eta}\left(F_{x, \delta}, F_{\bar{x}, \delta}\right) / 2} \\
= & e^{i j_{\eta}\left(F_{x}^{\alpha}-F_{\bar{x}}^{\alpha}\right)} e^{-i j_{\eta}\left(F_{x-\delta}^{\alpha}-F_{\bar{x}-\delta}^{\alpha}\right)} e^{i \alpha \sigma_{\eta}\left(F_{x}, F_{\bar{x}}\right)}
\end{aligned}
$$

noting that for sufficiently large $\delta$,

$$
\begin{gathered}
\sigma_{\eta}\left(F_{x}-F_{\bar{x}}, F_{x-\delta}-F_{\bar{x}-\delta}\right)=0 \\
\sigma_{\eta}\left(F_{x}, F_{\bar{x}}\right)=\sigma_{\eta}\left(F_{x, \delta}, F_{\bar{x}, \delta}\right) / 2 .
\end{gathered}
$$

If we take into account that $\omega$ is translation invariant and clustering

$$
\begin{aligned}
& \lim _{\delta \rightarrow \infty} \omega\left(e^{i j_{\eta}\left(F_{x}^{\alpha}-F_{\bar{x}}^{\alpha}\right)} e^{-i j_{\eta}\left(F_{x-\delta}^{\alpha}-F_{\bar{x}-\delta}^{\alpha}\right)}\right) \\
= & \omega\left(e^{i j_{\eta}\left(F_{x}^{\alpha}-F_{\bar{x}}^{\alpha}\right)}\right) \omega\left(e^{-i j_{\eta}\left(F_{x-\delta}^{\alpha}-F_{\bar{x}-\delta}^{\alpha}\right)}\right)=e^{-\alpha\left\langle F_{x}-F_{\bar{x}} \mid F_{x}-F_{\bar{x}}\right\rangle_{\eta}},
\end{aligned}
$$

we can conclude

$$
\omega\left(e^{i j_{\eta}(f)} e^{i j_{\eta}\left(F_{x}^{\alpha}\right)} e^{-i j_{\eta}\left(F_{\bar{x}}^{\alpha}\right)} e^{-i j_{\eta}(g)}\right)=\lim _{\delta \rightarrow \infty} \omega\left(e^{i j_{\eta}(f)} e^{i j_{\eta}\left(F_{x, \delta}^{\alpha}\right) / \sqrt{2}} e^{-i j_{\eta}\left(F_{\bar{x}, \delta}^{\alpha}\right) / \sqrt{2}} e^{-i j_{\eta}(g)}\right) .
$$


Now we can apply (2.7) and obtain

$$
\begin{aligned}
& \omega\left(\prod_{k} e^{i j_{\eta}\left(F_{x_{k}}^{\alpha}\right)}\right)=\lim _{\delta \rightarrow \infty} \omega\left(\prod_{k} e^{i j_{\eta}\left(F_{x_{k}, \delta}^{\alpha}\right) / \sqrt{2}}\right) \\
= & \lim _{\delta \rightarrow \infty} \exp \left\{-\frac{\alpha}{2}\left[\frac{1}{2} \sum_{k}\left\langle F_{x_{k}, \delta} \mid F_{x_{k}, \delta}\right\rangle_{\eta}+\sum_{k<m}\left\langle F_{x_{k}, \delta} \mid F_{x_{m}, \delta}\right\rangle_{\eta}\right]\right\} .
\end{aligned}
$$

We can evaluate the scalar products involved in (3.3):

$$
\begin{aligned}
& \left\langle F_{x_{k}, \delta} \mid F_{x_{m}, \delta}\right\rangle_{\eta}=\int d z \int_{-\delta+x_{k}}^{x_{k}} d y \int_{-\delta+x_{m}}^{x_{m}} d y^{\prime} \frac{\hat{h}_{\eta}(z)}{(2 \pi)^{2} \operatorname{sh}^{2}\left(y-y^{\prime}-z-i \varepsilon\right)} \\
= & \frac{1}{(2 \pi)^{2}} \int \hat{h}_{\eta}(z) d z \ln \frac{\operatorname{sh}^{2}\left(x_{k}-x_{m}-z-i \varepsilon\right)}{\operatorname{sh}\left(x_{k}-x_{m}+\delta-z-i \varepsilon\right) \operatorname{sh}\left(x_{k}-x_{m}-\delta-z-i \varepsilon\right)} .
\end{aligned}
$$

To get something finite for $\delta \rightarrow \infty$ we have to take operators of the form

$$
\prod e^{ \pm i j\left(F_{x_{k}}^{\alpha}\right)}:=\prod e^{i j\left(\bar{F}_{x_{k}}^{\alpha}\right)}
$$

where $\bar{F}_{x_{k}}^{\alpha}=s_{k} F_{x_{k}}^{\alpha}, s_{k}= \pm 1$. The individual expressions diverge with $\delta \rightarrow \infty$. If therefore the anyon contributions do not neutralize, i.e. if $\sum_{k} s_{k} \neq 0$, the expectation value (3.3) vanishes. On the other hand, if $\sum_{k} s_{k}=0$, we have as many positive contributions as negative ones, this means for the products $2 r+2 r(r-1)$ positive contributions and $2 r^{2}$ negative ones. Those that contain $\delta$ can be combined in pairs to

$$
\lim _{\delta \rightarrow \infty}\left[\ln \frac{\operatorname{sh}(x+\delta-i \varepsilon)}{\operatorname{sh}(y+\delta-i \varepsilon)}-\ln \frac{\operatorname{sh}(x-\delta-i \varepsilon)}{\operatorname{sh}(y-\delta-i \varepsilon)}\right]=\lim _{\delta \rightarrow \infty}\left(\ln \frac{e^{x+\delta}}{e^{y+\delta}}-\ln \frac{e^{\delta-x}}{e^{\delta-y}}\right)=0 .
$$

Therefore we remain in the limit $\delta \rightarrow \infty$ with

$$
\begin{gathered}
\omega\left(\prod_{k} e^{i j_{\eta}\left(\bar{F}_{x_{k}}^{\alpha}\right)}\right)=\left[c^{-2}(\eta)\right]^{n \alpha} \exp \left\{-\int d z \hat{h}_{\eta}(z) \alpha \ln \left(\prod_{k<m} s_{k} s_{m} \operatorname{sh}\left(x_{k}-x_{m}-z-i \varepsilon\right)\right)\right\} \\
c^{-1}(\eta)=\exp \left[\frac{1}{2} \int \hat{h}_{\eta}(z) \ln \operatorname{sh}(-z-i \varepsilon) d z\right] .
\end{gathered}
$$

the latter taking care for the ultraviolet divergence in $\Psi_{\alpha}$.

In terms of $\Psi$ 's this means that the expectation value of a product of $\Psi$ 's and $\Psi^{*}$ 's is different from zero only if there are as many $\Psi^{\prime}$ 's as $\Psi^{*}$ 's, equivalently — if the "total" statistic parameter of creation operators equals the one of annihilation operators, for instance $\left\langle\Psi_{1}^{*} \Psi_{1}^{*} \Psi_{4}\right\rangle \neq 0$, or otherwise that they lead to orthogonal sectors of the enlarged Hilbert space.

Performing the limit $\eta \rightarrow 0$, where $\hat{h}_{\eta}(x) \rightarrow \delta(x)$ we achieve our aim - construction of the local anyonic field operators. Note that these are now strong limits of the anyonic Weyl operators as discussed in [12, 13, 14]. 
The divergence for $\eta \rightarrow 0$ remains and determines the necessary renormalization of the operators $\Psi_{\alpha}$ [14

$$
\text { s- } \lim _{\eta \rightarrow 0} \int f(x) c(\eta) e^{i j_{\eta}\left(F_{x}^{\alpha}\right)} d x=\int f(x) \Psi_{\alpha}(x) d x .
$$

From (3.5), (3.6) we obtain for the $n$-point function

$$
\begin{aligned}
& \omega\left(\Psi_{\alpha}^{*}\left(x_{1}\right) \ldots \Psi_{\alpha}^{*}\left(x_{n}\right) \Psi_{\alpha}\left(y_{n}\right) \ldots \Psi_{\alpha}\left(y_{1}\right)\right) \\
= & \lim _{\eta \rightarrow 0}\left[c^{2}(\eta)\right]^{n \alpha} \omega\left(e^{-i j_{\eta}\left(F_{x_{1}}^{\alpha}\right)} \ldots e^{-i j_{\eta}\left(F_{x_{n}}^{\alpha}\right)} e^{i j_{\eta}\left(F_{y_{n}}^{\alpha}\right)} \ldots e^{i j_{\eta}\left(F_{y_{1}}^{\alpha}\right)}\right) \\
= & \frac{\prod_{k>l}\left(\operatorname{sh}\left(x_{k}-x_{l}-i \varepsilon\right)\right)^{\alpha} \prod_{k>l}\left(\operatorname{sh}\left(y_{k}-y_{l}-i \varepsilon\right)\right)^{\alpha}}{\prod_{k, l}\left(-2 \pi i \operatorname{sh}\left(x_{k}-y_{l}-i \varepsilon\right)\right)^{\alpha}} .
\end{aligned}
$$

The exact exchange relations are hidden in the factor $i^{\alpha}$ in (3.7) and we shall return to their detailed analysis later on.

For all $\alpha$ 's the two-point function (for $x>x^{\prime}$ and $\beta=\pi$ )

$$
\left\langle\Psi_{\alpha}^{*}(x) \Psi_{\alpha}\left(x^{\prime}\right)\right\rangle_{\beta}=\left\langle\Psi_{\alpha}(x) \Psi_{\alpha}^{*}\left(x^{\prime}\right)\right\rangle_{\beta}=\left(\frac{i}{2 \pi \operatorname{sh}\left(x-x^{\prime}\right)}\right)^{\alpha}=: S_{\alpha}\left(x-x^{\prime}\right)
$$

has the desired properties

(i) Hermiticity:

$$
S_{\alpha}^{*}(x)=S_{\alpha}(-x) \Longleftrightarrow\left\langle\Psi_{\alpha}^{*}(x) \Psi_{\alpha}\left(x^{\prime}\right)\right\rangle_{\beta}^{*}=\left\langle\Psi_{\alpha}^{*}\left(x^{\prime}\right) \Psi_{\alpha}(x)\right\rangle_{\beta} ;
$$

(ii) $\alpha$-commutativity:

$$
S_{\alpha}(-x)=e^{i \pi \alpha} S_{\alpha}(x) \Longleftrightarrow\left\langle\Psi_{\alpha}\left(x^{\prime}\right) \Psi_{\alpha}^{*}(x)\right\rangle_{\beta}=e^{i \pi \alpha}\left\langle\Psi_{\alpha}^{*}(x) \Psi_{\alpha}\left(x^{\prime}\right)\right\rangle_{\beta} ;
$$

(iii) KMS-property:

$$
S_{\alpha}(x)=S_{\alpha}(-x+i \pi) \Longleftrightarrow\left\langle\Psi_{\alpha}^{*}(x) \Psi_{\alpha}\left(x^{\prime}\right)\right\rangle_{\beta}=\left\langle\Psi_{\alpha}\left(x^{\prime}\right) \Psi_{\alpha}^{*}(x+i \pi)\right\rangle_{\beta} .
$$

For $\alpha=2$ and an arbitrary temperature $\beta^{-1}$ we get like for the $j^{\text {'s }}$

$$
\left\langle\Psi_{2}^{*}(x) \Psi_{2}\left(x^{\prime}\right)\right\rangle_{\beta}=-\frac{1}{\left(2 \beta \operatorname{sh} \frac{\pi\left(x-x^{\prime}-i \varepsilon\right)}{\beta}\right)^{2}},
$$

similarly, for $\alpha=3$ we get a different kind of fermions

$$
\left\langle\Psi_{3}^{*}(x) \Psi_{3}\left(x^{\prime}\right)\right\rangle_{\beta}=-\frac{i}{\left(2 \beta \operatorname{sh} \frac{\pi\left(x-x^{\prime}-i \varepsilon\right)}{\beta}\right)^{3}} .
$$

These fields, though locally (anti)commuting, are not canonical and this becomes transparent by analyzing temperature dependence and operator structure of their exchange relations. 


\section{The $\alpha$-commutator}

As a direct consequence of the Weyl relations (3.2) for the anyonic Weyl operators (1.3) and with (3.6) in mind, it follows that

$$
\Psi_{\alpha}^{*}(x) \Psi_{\alpha}(y)-e^{i \pi \alpha \operatorname{sgn}(x-y)} \Psi_{\alpha}(y) \Psi_{\alpha}^{*}(x)=0 \quad \text { for } x \neq y .
$$

It remains to calculate the distribution that emerges by bringing the field arguments together.

Evidently, the case $\alpha \in \mathbf{Z}$ plays a special role since we then deal with Fermi- or Bosecommutation relations. We first concentrate on $\alpha=1$. Then we observe

$$
\omega\left(\Psi^{*}\left(x_{1}\right) \ldots \Psi\left(y_{n}\right)\right)=\operatorname{Det} \omega\left(\Psi^{*}\left(x_{i}\right) \Psi\left(y_{k}\right)\right)=\operatorname{Det} w\left(x_{i}-y_{k}\right) .
$$

Proof: From Cauchy's determinant formula

$$
\operatorname{Det}\left(x_{i}-y_{k}\right)^{-1}=\frac{\prod_{i>k}\left(x_{i}-x_{k}\right) \prod_{i>k}\left(y_{i}-y_{k}\right)}{\prod_{i, k}\left(x_{i}-y_{k}\right)}
$$

one gets

$$
\operatorname{Det} \frac{\sqrt{x_{i} y_{j}}}{\left(x_{i}-y_{j}\right)}=\prod \sqrt{x_{i}} \prod \sqrt{y_{j}} \frac{\prod_{i>j}\left(x_{i}-x_{j}\right) \prod_{i>j}\left(y_{i}-y_{j}\right)}{\prod_{i, j}\left(x_{i}-y_{j}\right)}=\operatorname{Det} \frac{1}{\sqrt{x_{i} / y_{j}}-\sqrt{y_{j} / x_{i}}} .
$$

This corresponds by replacing $\sqrt{x}$ by $e^{x}, \sqrt{y}$ by $e^{y}$, to

$$
\operatorname{Det} \frac{1}{\operatorname{sh}\left(x_{i}-y_{k}-i \varepsilon\right)}=\frac{\prod_{i>k} \operatorname{sh}\left(x_{i}-x_{k}-i \varepsilon\right) \prod_{i>k} \operatorname{sh}\left(y_{i}-y_{k}-i \varepsilon\right)}{\prod_{i, k} \operatorname{sh}\left(x_{i}-y_{k}-i \varepsilon\right)} .
$$

\section{Theorem}

For $\alpha=1$ the renormalized fields $\Psi_{1}^{*}(x), \Psi_{1}(x)$ canonically anticommute

$$
\Psi_{1}^{*}(x) \Psi_{1}(y)+\Psi_{1}(y) \Psi_{1}^{*}(x)=\delta(x-y) .
$$

The state over the algebra of fermions is the quasifree state given by the two-point function

$$
\omega\left(\Psi_{1}^{*}(x) \Psi_{1}(y)\right)=\frac{i}{2 \pi \operatorname{sh}(x-y-i \varepsilon)} .
$$

It satisfies the KMS condition with respect to the shift for temperature $\beta=\pi$ (comp. (3.8)). For arbitrary temperature by scaling arguments it follows

$$
\omega_{\beta}\left(\Psi_{1}^{*}(x) \Psi_{1}(y)\right)=\frac{i}{2 \beta \operatorname{sh} \frac{\pi\left(x-x^{\prime}-i \varepsilon\right)}{\beta}},
$$


with the same commutation relations.

For arbitrary $\alpha$ we have to analyse in detail the expectation

$$
\begin{aligned}
& \omega_{\beta}\left(\Psi_{\alpha}^{*}\left(x_{1}\right) \ldots \Psi_{\alpha}\left(y_{n}\right)\right)=\frac{\prod_{k>l}\left[\operatorname{sh}\left(x_{k}-x_{l}-i \varepsilon\right)\right]^{\alpha} \prod_{k>l}\left[\operatorname{sh}\left(y_{k}-y_{l}-i \varepsilon\right)\right]^{\alpha}}{\prod_{i, j} \operatorname{sh}\left(x_{i}-y_{j}-i \varepsilon\right)^{\alpha}} \\
& =\left(\operatorname{Det} \operatorname{sh}^{-1}\left(x_{i}-y_{j}-i \varepsilon\right)\right)^{\alpha} .
\end{aligned}
$$

Evidently, for $\alpha \neq 1$ the state is determined again by the two-point function but not in a way that corresponds to a truncation. In order to deduce the commutation relations it is preferable to evaluate

$$
\begin{aligned}
& \int \omega\left(e^{i j\left(f_{1}\right)}\left[\Psi_{\alpha}^{*}(x) \Psi_{\alpha}(y)-e^{i \pi \alpha \operatorname{sgn}(x-y)} \Psi_{\alpha}(y) \Psi_{\alpha}^{*}(x)\right] e^{-i j\left(f_{2}\right)}\right) g_{1}(x) g_{2}(y) d x d y \\
= & \int \omega\left(e^{i j\left(f_{1}\right)} e^{-i j\left(f_{2}\right)}\right)\left(\frac{1}{\operatorname{sh}^{\alpha}(x-y-i \varepsilon)}-\frac{(-1)^{\alpha}}{\operatorname{sh}^{\alpha}(y-x-i \varepsilon)}\right)(i / 2 \pi)^{\alpha} \\
\times & e^{2 \pi \sqrt{\alpha}\left[\left\langle f_{1} \mid \Theta(x)\right\rangle-\left\langle f_{1} \mid \Theta(y)\right\rangle-\left\langle\Theta(x) \mid f_{2}\right\rangle+\left\langle\Theta(y) \mid f_{2}\right\rangle\right]} g_{1}(x) g_{2}(y) d x d y .
\end{aligned}
$$

Therefore the distribution to be considered is

$$
\lim _{\varepsilon \rightarrow 0^{+}}\left(\frac{1}{\operatorname{sh}^{\alpha}(x-i \varepsilon)}-\frac{1}{\operatorname{sh}^{\alpha}(x+i \varepsilon)}\right), \quad \alpha \notin \mathbf{Z}^{+} .
$$

This expression tends to 0 for $x \neq 0$. As for the singularity at $x=0$, after a partial integration one realizes that it suffices to assume $0<\alpha<1$ and to evaluate the remaining integral around the origin, so with $\operatorname{sh}(x-i \varepsilon)^{-\alpha} \sim(x-i \varepsilon)^{-\alpha}$

$$
\begin{aligned}
& \lim _{\varepsilon \rightarrow 0} \int_{-\delta}^{\delta}\left(\frac{1}{(x-i \varepsilon)^{\alpha}}-\frac{1}{(x+i \varepsilon)^{\alpha}}\right) f(x) d x \\
= & \lim _{\varepsilon \rightarrow 0} \int_{-\delta / \varepsilon}^{\delta / \varepsilon}\left(\frac{1}{(y-i)^{\alpha}}-\frac{1}{(y+i)^{\alpha}}\right) f(\varepsilon y) \varepsilon^{1-\alpha} d y=0
\end{aligned}
$$

Therefore for anyonic fields with noninteger statistic parameter the $\alpha$-commutator vanishes

$$
\Psi_{\alpha}^{*}(x) \Psi_{\alpha}(y)-e^{i \pi \alpha \operatorname{sgn}(x-y)} \Psi_{\alpha}(y) \Psi_{\alpha}^{*}(x)=0 \quad \alpha \notin \mathbf{Z}^{+} .
$$

For $\alpha$ integer we can perform again partial integration. This gives us for $\alpha=2$

$$
\lim _{\varepsilon \rightarrow 0}\left(\frac{1}{\operatorname{sh}^{2}(x-i \varepsilon)}-\frac{1}{\operatorname{sh}^{2}(x+i \varepsilon)}\right)=-2 \pi i \delta^{\prime}(x) .
$$


Therefore

$$
\begin{aligned}
& \int \omega\left(e^{i j\left(f_{1}\right)}\left[\Psi_{2}^{*}(x) \Psi_{2}(y)-\Psi_{2}(y) \Psi_{2}^{*}(x)\right] e^{-i j\left(f_{2}\right)} g_{1}(x) g_{2}(y)\right) d x d y \\
= & \frac{i}{2 \pi} \int \delta^{\prime}(x-y) \omega\left(e^{i j\left(f_{1}\right)} e^{-i j\left(f_{2}\right)}\right) e^{2 \pi \sqrt{2}\left[\left\langle f_{1} \mid \Theta(x)\right\rangle-\left\langle f_{1} \mid \Theta(y)\right\rangle-\left\langle\Theta(x) \mid f_{2}\right\rangle+\left\langle\Theta(y) \mid f_{2}\right\rangle\right]} \\
& \times g_{1}(x) g_{2}(y) d x d y+i \sqrt{2} \int g_{1}(x) g_{2}(x) \omega\left(e^{i j\left(f_{1}\right)} e^{-i j\left(f_{2}\right)}\right) d x
\end{aligned}
$$

or

$$
\left[\Psi_{2}^{*}(x), \Psi_{2}(y)\right]=\frac{i}{2 \pi} \delta^{\prime}(x-y)+i \sqrt{2} \delta(x-y) j(x) .
$$

This follows from comparing the contributions in the expectation value

$$
\delta^{\prime}(x-y) e^{\left[\left\langle f_{1} \mid \Theta(x)\right\rangle-\left\langle f_{1} \mid \Theta(y)\right\rangle-\left\langle\Theta(x) \mid f_{2}\right\rangle+\left\langle\Theta(y) \mid f_{2}\right\rangle\right]} \longrightarrow \delta(x-y)\left[\left\langle f_{1} \mid \delta(x)\right\rangle-\left\langle\delta(x) \mid f_{2}\right\rangle\right]
$$

with

$$
\omega\left(e^{i j\left(f_{1}\right)} j(x) e^{-i j\left(f_{2}\right)}\right)=\frac{d}{d \gamma} \omega\left(e^{i j\left(f_{1}\right)} e^{-i j\left(f_{2}\right)}\right) e^{\gamma\left[\left\langle f_{1} \mid \delta(x)\right\rangle-\left\langle\delta(x) \mid f_{2}\right\rangle\right]} .
$$

Thus the bosonic fields of the first level do not satisfy canonical commutation relations. Though the two-point functions look similar, for the initial bosonic algebra this was a two-point function of currents whereas now it is a two-point function of fields, that are not invariant under gauge automorphisms but are adjoint of each other.

For $\alpha=3$ again fermionic fields are obtained. Partial integration yields (we do not fix the temperature)

$$
\begin{aligned}
& \omega\left(e^{i j\left(f_{1}\right)}\left[\Psi_{3}^{*}(x) \Psi_{3}(y)+\Psi_{3}(y) \Psi_{3}^{*}(x)\right] e^{-i j\left(f_{2}\right)}\right) \\
= & -\frac{1}{8 \pi^{2}}\left(\delta^{\prime \prime}(x-y)-\frac{\pi^{2}}{\beta^{2}} \delta(x-y)\right) e^{2 \pi \sqrt{3}\left[\left\langle f_{1} \mid \Theta(x)\right\rangle-\left\langle f_{1} \mid \Theta(y)\right\rangle-\left\langle\Theta(x) \mid f_{2}\right\rangle+\left\langle\Theta(y) \mid f_{2}\right\rangle\right]} .
\end{aligned}
$$

Here the $\delta^{\prime \prime}(x-y)$ splits again in a distribution acting only on the smearing function of the fermion fields and additional terms, so that we can write the commutator as an operator-valued distribution

$$
\left[\Psi_{3}^{*}(x), \Psi_{3}(y)\right]_{+}=-\frac{1}{8 \pi^{2}}\left[\delta^{\prime \prime}(x-y)-2 \pi \sqrt{3} \delta^{\prime}(x-y) j(x)+12 \pi^{2} \delta(x-y) j^{2}(x)\right]+\frac{1}{8 \beta^{2}} \delta(x-y)
$$

or more precisely,

$$
\begin{aligned}
{\left[\Psi_{3}^{*}(f), \Psi_{3}(g)\right]_{+} } & =-\frac{1}{8 \pi^{2}} \int d x\left\{\frac{d^{2}}{d x^{2}} f(x) g(x)-2 \pi \sqrt{3} j(x)\left(\frac{d}{d x} f(x) g(x)\right)\right. \\
& \left.+12 \pi^{2} f(x) g(x) j^{2}(x)-(\pi / \beta)^{2} f(x) g(x)\right\}
\end{aligned}
$$

Thus we are faced with a new feature: the commutation relations become temperature dependent, though they remain local. The operators $\Psi_{3}(f)$ are obviously not bounded. 
The fermions of the third level form a subalgebra of the whole fermionic algebra (the fermions of the first level). This demonstrates that representations corresponding to different temperatures are inequivalent not only globally, but already also locally.

\section{Back to the current algebra}

The current algebra can be considered as the subalgebra of any anyonic algebra $\left\{\Psi_{\alpha}(x)\right\}^{\prime \prime}$ that is invariant under the gauge automorphism $\gamma_{s} \Psi_{\alpha}(x)=e^{i \alpha s} \Psi_{\alpha}(x)$. Furthermore, the local fields $j(x)$ can be directly constructed out of the anyonic fields $\Psi_{\alpha}(x)$. Starting with the fermions $\Psi_{1}(x)$ this procedure is well known [1, 2]. In fact, $\Psi^{*}(x) \Psi(x)$ is at best a quadratic form and by point splitting it becomes the operator-valued distribution $\Psi^{*}(x+\varepsilon) \Psi(x-\varepsilon)$. We can relate this to the bounded operator $e^{i j\left(f_{x, \varepsilon}\right)}$ with $f_{x, \varepsilon}(y)=1$ for $x-\varepsilon<y<x+\varepsilon$, otherwise 0 . But then we produce by the sharp edge of $f$ an ultraviolet problem with diverging expectation values. To start with a well defined expression with a zero expectation value we consider

$$
j(x)=\lim _{\substack{f x, \varepsilon(x)=1 \\ f_{x, \varepsilon}(y) d y \rightarrow \underset{\varepsilon \rightarrow 0}{\rightarrow} 0}} \frac{e^{i \alpha j\left(f_{x, \varepsilon}\right)}-e^{-i \alpha j\left(f_{x, \varepsilon}\right)}}{2 i \alpha \int f_{x, \varepsilon}(y) d y} .
$$

Now we choose

$$
f_{x, \varepsilon}(y):=\left\{\begin{array}{cl}
0 & \text { for } y<x-\varepsilon, y>x+\varepsilon \\
1-\left|\frac{x-y}{\varepsilon}\right| & \text { for } x-\varepsilon<y<x+\varepsilon
\end{array}\right.
$$

This can be interpreted as a smearing in the sense of (2.4) so that

$$
e^{i \alpha j\left(f_{x, \varepsilon}\right)}=\Psi_{\alpha, \varepsilon}^{*}(x+\varepsilon) \Psi_{\alpha, \varepsilon}(x-\varepsilon),
$$

whereas

$$
e^{-i \alpha j\left(f_{x, \varepsilon}\right)}=\Psi_{\alpha, \varepsilon}^{*}(x-\varepsilon) \Psi_{\alpha, \varepsilon}(x+\varepsilon)
$$

and $\int f_{x, \varepsilon}(y) d y$ corresponds to the renormalization factor needed to pass from the anyonic Weyl operators to the anyonic fields. Of course, $j(x)$ is not an operator but has to be smeared by a suitable $f$. Then (4.3) becomes a strong resolvent limit. In a sense we thus provide a replacement of the familiar expression

$$
j(x)=: \Psi^{*}(x) \Psi(x):
$$

that has been used to construct a bosonic current algebra with Schwinger commutation relations out of the fermions. In fact, since we obtain the anyonic fields as limit of anyonic Weyl operators that are not quadratic in the Fermi field, the current emerges out of a kind of a Dirac sea filling. This procedure is state dependent and the states under consideration are not locally normal, therefore the structure of the Dirac sea, the higher-level fields exhibit a nontrivial temperature dependence. Nevertheless, the structure of the currents defined by (4.3) is $\alpha$ and $\beta$ independent. 


\section{The Luttinger model}

The Luttinger model has been designed to describe a one-dimensional interacting electron system. The model spectrum in the ground state consists of plasmons with a well-defined energy [5]. The essential input in these considerations is the fact that the free part of the Hamiltonian makes a Bogoliubov transformation necessary, so that the Bose operators, which replace (appropriately) the products of fermionic creation and annihilation operators $a^{*}(x) a(x)$, satisfy the Schwinger commutation relations (1.2).

Several interaction potentials can be considered

1. $H=H_{0}+\lambda \int j_{1}(x) v(x-y) j_{2}(y)$;

2. $H=H_{0}+\lambda \int\left(j_{1}(x)+j_{2}(x)\right) v(x-y)\left(j_{1}(y)+y_{2}(y)\right)$;

3. $H=H_{0}+\lambda \int j_{1}(x) v(x-y) j_{1}(y)$.

With $\rho_{i}(p)=\int j_{i}(x) e^{i p x} d p,(1.2)$ leads to

1. $\left(\ddot{\rho}_{1}(p)+\ddot{\rho}_{2}(p)\right)=-p^{2}\left(1-\lambda^{2} \tilde{v}^{2}(p)\right)\left(\rho_{1}(p)+\rho_{2}(p)\right)$;

2. $\left(\ddot{\rho}_{1}(p) \pm \ddot{\rho}_{2}(p)\right)=\left(p^{2}+2 \lambda p \tilde{v}(p)\right)\left(\rho_{1}(p) \pm \rho_{2}(p)\right)$;

3. $\dot{\rho}(p)=p(1+\lambda \tilde{v}(p)) \rho(p)$.

In all cases we obtain a quasifree evolution for the currents. There exist the corresponding KMS-states, provided they are positiv definite (which might bring in some restrictions on the coupling constant). With $\tau_{t} j(f)=j\left(e^{i \varepsilon(p) t} f\right)$,

$$
\begin{aligned}
\omega(j(f) j(g)) & =\langle f \mid g\rangle_{\beta, v}=\int f(x) g(y) w(x-y)_{\beta, v} \\
& =\int_{-\infty}^{\infty} \frac{p}{1-e^{-\beta \varepsilon(p)}} \tilde{f}(p) \tilde{g}(-p) d p \\
& =\int_{0}^{\infty} \frac{p}{1-e^{-\beta \varepsilon(p)}}\left(\tilde{f}(p) \tilde{g}(-p)+\tilde{f}(-p) \tilde{g}(p) e^{-\beta \varepsilon(p)}\right) .
\end{aligned}
$$

As for the free evolution, the state can be extended to the anyonic Weyl operators as in (3.3), resulting in a KMS-state for the extended algebra. For sufficiently smooth short-range potentials

$$
\lim _{p \rightarrow \infty} \frac{\varepsilon(p)}{p}=1, \quad \lim _{x \rightarrow \pm \infty} \frac{w(x)_{\beta, v}}{w(x)_{\beta}}=1 .
$$


Therefore the infrared behaviour in $\left\langle F_{x, \delta} \mid F_{\bar{x}, \delta}\right\rangle$ for $\delta \rightarrow \infty$ can be controled as for the free case. Since $\tilde{w}_{\beta}(p)-\tilde{w}_{\beta, v}(p) \in \mathcal{S}$ also the ultraviolet behaviour is unchanged. It is essential again that the bosonic one-particle Hamiltonian is positiv definite and does not produce an additional singularity at some $p \neq 0$. Also here the $n$-point functions become products of two-point functions (4.2). However, already for the $\alpha=1$ fermions $\varepsilon(p) \neq p$ and they do not allow the decomposition (4.1), so that the state over the fermionic algebra is not quasifree any more. Nevertheless, the resulting fermions satisfy canonical anticommutation relations because the leading singularity in $w_{\beta, v}(x)$ coincides with that of $w_{\beta}(x)$ and determines the commutation relations

$$
\omega_{\beta, v}\left(e^{i j\left(f_{1}\right)}\left[\Psi^{*}(x) \Psi(y)+\Psi(y) \Psi^{*}(x)\right] e^{-i j\left(f_{2}\right)}\right)=\omega_{\beta, v}\left(e^{i j\left(f_{1}\right)} e^{-i j\left(f_{2}\right)}\right) \delta(x-y) .
$$

This shows that $[., .]_{+}$is a $c$-number and that $\omega_{\beta, v}$ is in fact a KMS-state for fermions with a point pair interaction, provided we accept that it results from a regularization procedure involving both delocalization and filling of the Dirac sea. Due to the fact that only the leading singularity remains unchanged, one should be aware of the indispensable

dependence of the exchange relations of the anyonic fields (especially of the higher-level Bose- and Fermi- fields) both on the temperature and interaction.

\section{Conclusions}

We have represented the two-dimensional chiral anyonic field $\Psi_{\alpha}$ as an operator in a Hilbert space and have studied its exchange relations and its thermal correlation functions. The latter are still determined by the two-point function

$$
\omega_{\beta}\left(\Psi_{\alpha}^{*}(x) \Psi_{\alpha}(y)\right)=\left(\frac{i}{2 \beta \operatorname{sh} \frac{\pi\left(x-x^{\prime}-i \varepsilon\right)}{\beta}}\right)^{\alpha},
$$

however not via simple truncation, but as

$$
\omega_{\beta}\left(\Psi_{\alpha}^{*}\left(x_{1}\right) \ldots \Psi_{\alpha}\left(y_{n}\right)\right)=\left(\operatorname{Det} \frac{i}{2 \beta \operatorname{sh} \frac{\pi\left(x_{i}-y_{k}-i \varepsilon\right)}{\beta}}\right)^{\alpha} .
$$

For $\alpha$ not integer the $\alpha$-commutator vanishes, for $\alpha$ odd the fields obtained are fermions and for $\alpha$ even they are bosons. However, for $\alpha>1$ the fields still being local, are not canonical, as it follows from the operator structure of their (anti)commutators. Moreover, already the first noncanonical fermions are not bounded and their commutation relations exhibit essential temperature dependence, so representations corresponding to different temperatures are not locally normal. Along this line of considerations, in the Luttinger model we have found an example of a not quasi-free KMS-state. 


\section{Acknowledgments}

We are grateful to A. Alekseev and I. Todorov for stimulating our interest into the problem and to H.J. Borchers, E. Lieb and B. Schroer for suggestive discussions .

N.I. thanks the International Erwin Schrödinger Institute for Mathematical Physics where the research has been performed, for hospitality and financial support. This work has been supported in part also by "Fonds zur Förderung der wissenschaftlichen Forschung in Österreich" under grant P11287-PHY.

\section{References}

[1] Jordan P 1935 Z. Phys. 93 464; 1936 ibid. 98 759; 1936 ibid. 99109

[2] Born M and Nagendra-Nath N 1936 Proc. Ind. Acad. Sci. 3318

[3] Ilieva N and Thirring W 1999 Eur. Phys. J. C6 705

[4] Ilieva N and Thirring W 1999 Problems of QFT ed B.M. Barbashov et al (Dubna, Russia: JINR Press, E2-99-35) p 167

[5] Mattis D C and Lieb E 1965 J. Math. Phys. 6304

[6] Mandelstam S 1975 Phys. Rev. D11 3026

[7] Doplicher S, Haag R and Roberts J 1969 Commun. Math. Phys. 15173

[8] Buchholz D, Doplicher S, Longo R and Roberts J 1993 Commun. Math. Phys. 155 123

[9] Ilieva N and Narnhofer H 1996 ÖAW Sitzungsber. II 20513

[10] Heisenberg W 1954 Z. Naturforsch. 9a 292

[11] Liguori A, Mintchev M and Pilo L 2000 Nucl. Phys. B569 577

[12] Carey A L and Ruijsenaars S N M 1987 Acta Appl. Math. 101

[13] Acerbi F, Morchio G and Strocchi F 1992 Lett. Math. Phys. 26 13; 1993 ibid. 271

[14] Ilieva N and Thirring W 1999 Theor. Math. Phys. 1211294 